\title{
Correlation of Coronary Calcium Scores with Growth Differentiation Factor-15 Levels in Patients with Coronary Artery Disease
}

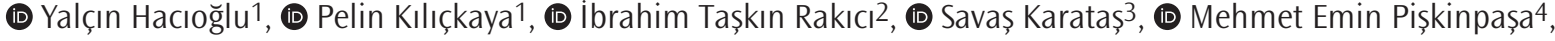 \\ (D) Turgut Karabağ5 \\ 1University of Health Sciences Turkey, İstanbul Training and Research Hospital, Clinic of Family Medicine, İstanbul, Turkey \\ 2University of Health Sciences Turkey, İstanbul Training and Research Hospital, Clinic of Radiology, İstanbul, Turkey \\ 3University of Health Sciences Turkey, İstanbul Training and Research Hospital, Clinic of Endocrinology and Metabolism, İstanbul, Turkey \\ ${ }^{4}$ University of Health Sciences Turkey, İstanbul Training and Research Hospital, Clinic of Internal Medicine, İstanbul, Turkey \\ 5 University of Health Sciences Turkey, İstanbul Training and Research Hospital, Clinic of Cardiology, İstanbul, Turkey
}

\begin{abstract}
Introduction: As noted in the guidelines, the efficacy of coronary artery calcium score (CACS) is remarkable in the intermediate-risk group for coronary artery disease; however, it exposes the patients to radiation. In this study, we aimed to investigate whether growth differentiation factor-15 (GDF-15) could be an alternative to CACS in patients with traditional cardiovascular risk factors.

Methods: In 2018, 86 volunteer patients (female: $n=36$; male: $n=50$ ) aged 25-85 years were included in the study among all patients whose CACS measurements were made in the radiology clinic. In our hospital, CACS images are obtained using 64-slice computed tomography. Serum GDF-15 levels were measured from venous blood. Participants were divided into two groups as zero and high $(>0)$ according to the CACS. SPSS 21.0 was used for the statistical analysis.

Results: Participants were compared with zero (female: $n=16$; male: $n=17$; mean age: 53.93 years) and high (>0) (female: 20, male: 33; mean age: 58.2 years) CACS groups. Leukocytes, GDF-15 levels, and monocyte/high-density lipoprotein-cholesterol (HDL-C) ratio were significantly higher in the CACS group $(p<0.05)$. Significant positive correlations were found between CACS and GDF-15 ( $r s=0.21)$, monocyte/HDL-C ratio ( $r s=0.29)$, and platelet/lymphocyte ratio ( $r s=0.21)(p<0.05)$.

Conclusion: In this study, GDF-15 and inflammatory markers were positively correlated with CACS. A significant difference was found in GDF-15 between patients with and without critical stenosis. Therefore, we can predict that the prognostic value of GDF-15 will be higher, especially in patients with critical stenosis. In this respect, studies with larger samples are needed.
\end{abstract}

Keywords: Coronary artery disease, coronary artery calcium score, growth differentiation factor 15

\section{Introduction}

Coronary artery disease (CAD) remains a significant public health problem in developed countries. Conditions such as industrial nutrition, sedentary life, smoking, and genetic and environmental factors that emerged after the industrial revolution negatively affect coronary vascular health.

The coronary artery calcium score (CACS) is an important prognostic marker in the moderate-risk group of CAD (1). Guidelines recommend its use for asymptomatic moderate-risk individuals with a $10-20 \%$ risk of cardiovascular (CV) events at 10 years, according to the Framingham Risk score (FRS) (1). However, the disadvantage of scanning is that healthy people are exposed to radiation. Thus, it is vital to achieve the same effect with radiation-free methods to protect healthy individuals.
Growth differentiation factor-15 (GDF-15) is a distant member of the transforming growth factor-beta superfamily, whose expression is increased under cellular stress. GDF-15 regulates biological processes involving multiple cell functions, differentiation, and regeneration of tissues (2). In CV events, GDF-15 is associated with increased mortality in people with heart failure, coronary heart disease (CHD), cardiac hypertrophy, and acute coronary syndrome (3).

In this study, we investigated whether GDF-15 could be an alternative to CACS in patients with traditional CV risk factors.

\section{Methods}

Between March and November 2018, 86 volunteer patients aged 25-85 years (female: $n=36$, male: $n=50$; mean age: 56.67 years) who applied 
to the radiology clinic for CACS screening due to traditional CV risk factors were included in the study. Patients with pregnancy, acute infection, acute vascular event, malignancy, known genetic disease, rheumatological disease, chronic renal failure, uncontrolled thyroid function tests, old stents, and inadequate cooperation and orientation were excluded from the study. All participants' anamnesis was taken, and detailed physical examinations were performed.

The approval form the University of Health Sciences Turkey, İstanbul Training and Research Hospital Local Ethics Committee was obtained (approval number: 1197, date: 09.03.2018). All patients have read and signed informed consent forms before participation.

Images were obtained on a 64-slice CT (Toshiba Aquilion system, Tokyo, Japan) with $400 \mathrm{~mm}$ rotation time and a $1 \mathrm{~mm}$ reconstruction device capable of descending to 0.5 sections. An automatic dose modulation system was used in the examinations. Images obtained without intravenous use of drugs using the electrocardiogram trigger feature with a $3 \mathrm{~mm}$ slice thickness were evaluated on the Aqua 3D workstation (Toshiba, Tokyo, Japan). Lesions with density >130 HU were marked and classified according to the Agatston technique. Percentile calculation for CACS values was performed with https://www.mesa-nhlbi.org/calcium/ input.aspx.

According to the CACS, elective coronary angiography (CAG) was performed in the indicated patients, and $\geq 50 \%$ stenosis in a single vessel was considered critical stenosis.

Biochemistry and hemogram parameters were measured from the venous blood sample after $8 \mathrm{~h}$ of fasting. Monocyte/high-density lipoprotein-cholesterol (HDL-C) and platelet/lymphocyte ratios, which are used as indicators of oxidative stress and systemic inflammation, were calculated from the obtained hemogram parameters. Laboratory results obtained in the routine follow-up of patients were used, and no additional expense was incurred.

In addition, extra venous blood was taken into a biochemistry tube and centrifugated. It was stored in a $-80{ }^{\circ} \mathrm{C}$ cabinet until the end of the study, and serum GDF-15 levels were then measured by using the sandwich enzyme-linked immunosorbent assay (human GDF-15 ELISA Kit, Elabscience, Wuhan, China). This kit recognizes human GDF-15 in samples. It has a detection range of $23.44-1500 \mathrm{pg} / \mathrm{mL}$, sensitivity of $14.06 \mathrm{pg} / \mathrm{mL}$, and repeatability/coefficient of variation $<10 \%$.

\section{Statistical Analysis}

Power analysis was performed, and a sample size of 90 was determined (alpha: 0.05, power: 0.8) (4). The suitability of the quantitative variables to a normal distribution was evaluated using the Shapiro-Wilk test. Participants were evaluated as zero and high (>0) groups according to the CACS, and data were compared between the two groups. A 50\% stenosis in CAG was accepted as critical stenosis. All participants who underwent CAG were regrouped according to the presence of critical stenosis, and available data were compared between the two groups. Finally, the correlations of CACS and GDF-15 with each other and with other parameters were analyzed.
In descriptive statistics, the mean and standard deviation were used in the expression of quantitative variables. Categorical variables are expressed by frequency and valid percentage. The $p$-value was accepted as $<0.05$ for significance. The results were evaluated at the $95 \%$ confidence interval. Data evaluation and analysis were performed using SPSS version 21 (IBM Corp., Armonk, NY, USA).

\section{Results}

All participants in the study (female: $n=36$; male: $n=50$; mean age: 56.67 years) were divided into two groups. Group 1 had zero CACS (female: $\mathrm{n}=16$; male: $\mathrm{n}=17$; mean age: 53.93 years), and group 2 had high $(>0)$ CACS (female: $n=20$; male: $n=33$; mean age: 58.2 years). Leukocytes, GDF-15, and monocytes/HDL-C ratio of the high CACS group were higher on average, and these differences were significant $(p<0.05)$ (Table 1). $\mathrm{C}$-reactive protein and platelet/lymphocyte ratio were on average higher in the high CACS group. However, these between-group differences were not significant $(p>0.05)$ (Table 1).

According to the CACS results, elective CAG was performed as necessary, and stenosis $\geq 50 \%$ in a single vessel was considered critical stenosis. Participants with critical stenosis (female: $n=9$; male: $n=18$; mean age: 64.9 years) and those without critical stenosis (female: $n=26$; male: $\mathrm{n}=33$; mean age: 52.8 years) were compared. CACS, percentile values, GDF-15, monocyte/HDL-C ratio, and thrombocyte/lymphocyte ratio were higher in patients with critical stenosis, and this difference was significant between the two groups $(p<0.05)$ (Table 2$)$.

The correlation of CACS with other parameters was examined. A significantly positive correlation with GDF-15 ( $r s=0.21$ ), monocyte/ HDL-C ( $r s=0.29$ ), and platelet/lymphocyte ratio ( $r s=0.21$ ) ratios was found $(p<0.05)$ (Table 3). When the correlation of GDF-15 with other parameters was examined, a significant positive correlation with the percentile values was noted ( $r s=0.34, p=0.001$ ). However, no significant correlation was found between GDF-15 and monocyte/HDL-C and thrombocyte/lymphocyte ratios ( $p>0.05$ ) (Table 3).

The receiver operating characteristics analysis was performed for CACS and GDF-15, which determine critical coronary artery stenosis. The areas

\begin{tabular}{|c|c|c|c|}
\hline & $\begin{array}{l}\text { CACS }=0 \\
(n=33)\end{array}$ & $\begin{array}{l}\text { CACS }>0 \\
(n=53)\end{array}$ & p-value \\
\hline Age (years) & $53.93 \pm 11.42$ & $58.2 \pm 11.31$ & $p=0.09 T$ \\
\hline \multicolumn{4}{|l|}{ Gender } \\
\hline Female & $16(48.5)$ & $20(37.5)$ & \multirow{2}{*}{$p=1.0 \chi^{2}$} \\
\hline Male & $17(51.5)$ & $33(62.2)$ & \\
\hline CRP (mg/L) & $4.21 \pm 6.21$ & $9.13 \pm 11.29$ & $p=0.21 T$ \\
\hline $\mathrm{WBC}\left(\times 10^{3} / \mu \mathrm{L}\right)$ & $7.28 \pm 1.38$ & $8.18 \pm 2.26$ & $p=0.03 \mathrm{~T}$ \\
\hline GDF-15 (pg/mL) & $1611 \pm 712$ & $1925 \pm 595$ & $p=0.03 \mathrm{~T}$ \\
\hline Monocytes/HDL-C & $0.009 \pm 0.006$ & $0.01 \pm 0.007$ & $p=0.01 T$ \\
\hline Platelet/lymphocyte & $105.59 \pm 52.98$ & $115.97 \pm 62.60$ & $p=0.43 T$ \\
\hline
\end{tabular}

Student's t-test $(\mathrm{T})$ : mean \pm standard deviation, chi-square test $\left(\chi^{2}\right): \mathrm{n}(\%$ valid). CACS: Coronary artery calcium score, CRP: C-reactive protein, GDF-15: growth differentiation factor-15, HDL-C: high-density lipoprotein-cholesterol, WBC: white blood cells 
under the curve were 0.834 for CACS (sensitivity, 96\%; specificity, 66.1\%; cut-off, 69; $\mathrm{p}<0.01$ ) and 0.389 for GDF-15 (sensitivity, 45.8\%; specificity, 78.9\%; cut-off, 1608.7; $p=0.11$ ) (Figure 1).

\section{Discussion}

\section{CAD and CACS}

Atherosclerosis is the main pathogenetic cause of CAD, which is a significant cause of mortality and morbidity. The atherosclerosis process begins early in life and progresses slowly (5). Vascular calcification appears as calcification or ossification at the base of advanced atherosclerotic lesions and has long been considered a natural consequence of aging. However, this is not an inevitable end and is an active pathological process (6).

CAD may be asymptomatic for a long time. Thus, it is critical to develop a screening strategy to identify those at moderate- and high-risk individuals without symptoms and to protect them from potential death and disability (7). A strong relationship was found between CV events and CACS in asymptomatic cases (8). For example, in 2004, Greenland et al. (9) evaluated whether CACS has additional prognostic significance for CHD and followed the participants for 7 years. After the study, CACS estimates individuals with a 10 -year risk $>10 \%$ according to FRS (9). In another study, $69 \%$ of the participants constituted a severe-risk group when assessed with FRS alone. However, this rate increased to $77 \%$ in the combined evaluation using FRS and CACS (10). In 2010, Erbel et al. (11) stated that adding CACS to traditional risk scoring improves the prediction of coronary death or non-fatal myocardial infarction.

\begin{tabular}{|c|c|c|c|}
\hline & $\begin{array}{l}\text { Non-critical } \\
\text { stenosis } \\
(n=59)\end{array}$ & $\begin{array}{l}\text { Critical } \\
\text { stenosis } \\
(n=27)\end{array}$ & p-value $(T)$ \\
\hline CACS & $222 \pm 438$ & $1074 \pm 1697$ & $p=0.01$ \\
\hline Percentile & $48.6 \pm 42.5$ & $87.70 \pm 13.47$ & $\mathrm{p}<0.001$ \\
\hline GDF-15 (pg/mL) & $1717 \pm 644$ & $2034 \pm 683$ & $p=0.04$ \\
\hline Monocyte/HDL-C & $0.010 \pm 0.007$ & $0.014 \pm 0.006$ & $p=0.02$ \\
\hline Platelet/lymphocyte & $100.5 \pm 54.3$ & $136.9 \pm 62$ & $p=0.007$ \\
\hline \multicolumn{4}{|c|}{ Student's t-test $(\mathrm{T})$ : mean \pm standard deviation. } \\
\hline
\end{tabular}

CACS is highly specific for coronary atherosclerosis. The sensitivity of CACS for obstructive CAD has been reported to be between $88 \%$ and $100 \%$. Moreover, the amount of calcium was associated with the total size of the atherosclerotic plaque (12). An autopsy study reported that coronary artery plaques show a positive correlation with CACS (13). An intracoronary ultrasound study also revealed that CACS is related to the location and grade of atherosclerotic plaques (14). As a result, the guidelines stated that CACS should be considered in CV risk assessment of moderate-risk individuals without symptoms (level of evidence class Ila, level B) (1). As regards cost-effectiveness, CACS is better than existing screening methods for intermediate-risk men (15).

CACS was first described by Agatston et al. (8) using ultrafast CT in 1990. It is used to calculate hyperdense lesions measured at $\geq 130 \mathrm{HU}$ in the axial plane tomography sections. Individual CAD risk is determined according to the Agatston classification (16). However, some ethical issues prevail with the widespread use of CACS. CACS scanning exposes the patients to ionizing radiation and some people indicated for screening are healthy individuals (7). The effective dose for CACS using CT is 10 times the effective dose (0.7-1.8 mSv) for chest X-ray imaging (17). However, a

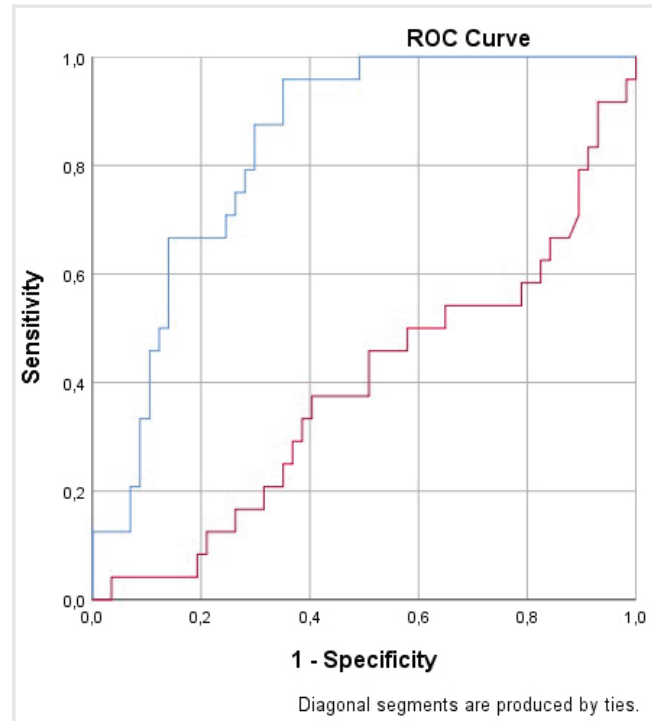

Figure 1. ROC analysis of CACS and GDF-15 to determine critical CAS AUC: CACS $=0.834$, GDF-15=0.389, CACS: Coronary artery calcium score, GDF-15: growth differentiation factor-15, CAS: coronary artery stenosis, AUC: area under the curve, ROC: receiver operating characteristics

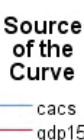

Table 3. Correlation levels

\section{With CACS}

GDF-15 (pg/mL)

Monocytes/HDL-C

Platelet/lymphocyte

With GDF-15

Percentile

Monocytes/HDL-C

Platelet/lymphocyte

$\rho$ : Pearson correlation analysis, s: Spearman's Rho correlation analysis.

CACS: coronary artery calcium score, GDF-15: growth differentiation factor 15, HDL-C: high-density lipoprotein-cholesterol

\begin{tabular}{|l|l|l|l|l|l|}
\hline $\mathbf{n}$ & r value & $\mathbf{r}^{\mathbf{2}}$ & $\mathbf{p}$ value $(\rho)$ & $\mathbf{r}$ s value & $\mathbf{p}$ value $(\mathbf{s})$ \\
\hline 86 & 0.18 & 0.032 & $p=0.08$ & 0.21 & $\mathbf{p}=\mathbf{0 . 0 4}$ \\
\hline 86 & 0.22 & 0.048 & $p=0.03$ & 0.29 & $\mathbf{p}=\mathbf{0 . 0 0 5}$ \\
\hline 86 & 0.15 & 0.022 & $p=0.16$ & 0.21 & $\mathbf{p}=\mathbf{0 . 0 4}$ \\
\hline & & & & & \\
\hline 86 & 0.33 & 0.108 & $p=0.002$ & 0.34 & $\mathbf{p}=\mathbf{0 . 0 0 1}$ \\
\hline 86 & -0.03 & $<0.001$ & $p=0.72$ & -0.09 & $p=0.37$ \\
\hline 86 & -0.08 & 0.006 & $p=0.43$ & -0.11 & $p=0.3$ \\
\hline
\end{tabular}


single moderate exposure ( $2.3 \mathrm{mSv}$ ) at age 40 years causes cancer, with a rate of 28 per 100,000 in women and 9 per 100,000 in men (18).

\section{GDF-15 and CV Diseases}

GDF-15 is one of the markers associated with cardiometabolic syndrome (19). GDF-15 regulates biological processes, including multiple cell functions, differentiation, and tissue regeneration (2). It facilitates the apoptosis of tumor cells and acts as a suppressor on metastasis (20). GDF-15 levels increase physiologically with age and during pregnancy $(19,21)$. GDF-15 is secreted from active macrophages in human vascular smooth muscle cells, human endothelial cells, and human adipocytes, which are stimulated by proinflammatory cytokines (22). Atherosclerotic plaques also express GDF-15. Some other conditions associated with high GDF-15 levels include smoking, type 2 diabetes mellitus, metabolic syndrome, sepsis, and chronic inflammatory conditions such as rheumatoid arthritis, chronic kidney disease, anemia, solid cancers, and cachexia (23).

In CV events, studies have shown that individuals with CV risk factors or a history of CV disease have higher levels of GDF-15 than those without (24). A study reported that GDF-15 is associated with coronary artery calcification and atherosclerotic plaque burden in the coronary artery (23). In the AtheroGene study, GDF-15 was associated with CHD mortality, left ventricular ejection fraction, and number of diseased vessels independent of conventional CV risk factors (25). Furthermore, in the "Heart and Soul" study, all-cause mortality and fatal/non-fatal CV events were independently associated with GDF-15 levels (26). Therefore, high GDF-15 levels may indicate occult CV disease in healthy individuals (27). Another critical point is that a study identified GDF-15 as a cardioprotective agent (28). In a study of CHD, the animal model has shown that GDF-15 has anti-inflammatory, anti-hypertrophic, and anti-apoptotic properties that may have a protective role and improve tissue repair (3).

\section{GDF-15 and CACS}

Both CACS and GDF-15 have prognostic significance for CAD independent of traditional CV risk factors. Studies have also examined both. For example, in 2017, Martinez et al. (29), examined the correlation of GDF-15 levels and subclinical atherosclerosis with CACS in patients with stable chronic obstructive pulmonary disease. GDF-15 was associated with high CACS at high tertile compared with low tertile (29). The "Dallas Heart Study," which examined the relationship between GDF15 and subclinical atherosclerosis, noted that GDF-15 is independently associated with subclinical coronary atherosclerosis, and the potential role of GDF-15 deserves further evaluation (22).

In our study, GDF-15 was associated with coronary atherosclerosis as determined by the CACS, similar to reports in some studies. When the participants were compared according to their CACS, a significant difference in GDF-15 was found. In addition, a positive correlation was found between CACS and GDF-15. According to CAG results, those with and without critical stenosis were compared, and a significant difference was found in GDF-15. In this respect, the prognostic value of GDF-15 will be higher, especially in patients with critical stenosis. Similarly, the monocyte/HDL-C ratio, an inflammatory marker, is also associated with coronary atherosclerosis in our study. A significant difference was found between the groups in terms of monocyte/HDL-C ratio in the evaluation with both CACS and critical stenosis.

\section{Study Limitations}

The sample size could not be expanded because of the limited number of kits and the completion of our study in a certain time. Our patient population consists of people with low socioeconomic status given our hospital's location, which reduces the diversity of our sample. Moreover, our relatively limited population reduces the statistical power. All these limit the generalization of our results.

\section{Conclusion}

The development of nonionizing and non-invasive methods is essential in identifying individuals at moderate-risk for CAD. GDF-15 and inflammatory markers appear suitable for this purpose. After our study, GDF-15 levels and monocyte/HDL-C ratio were higher in individuals with high CACS, and a significantly positive correlation was found between CACS and monocyte/HDL-C ratios and GDF-15 levels. In critical stenosis, both monocyte/HDL-C ratios and GDF-15 levels were significantly higher than non-critical stenosis. Therefore, we can predict that the prognostic value of GDF-15 will be higher, especially in those with critical stenosis. In this respect, studies with larger samples are needed.

Ethics Committee Approval: The approval form the University of Health Sciences Turkey, İstanbul Training and Research Hospital Local Ethics Committee was obtained (approval number: 1197, date: 09.03.2018).

Informed Consent: All patients have read and signed informed consent forms before participation.

Peer-review: Externally peer-reviewed.

Authorship Contributions: Surgical and Medical Practices - Y.H., I.T.R., T.K.; Concept - Y.H., S.K.; Design - M.E.P., T.K.; Data Collection or Processing - I.T.R., S.K., M.E.P.; Analysis or Interpretation - P.K., S.K., T.K.; Literature Search - Y.H., P.K., I.T.R.; Writing - P.K., M.E.P.

Conflict of Interest: No conflict of interest was declared by the authors.

Financial Disclosure: The authors declared that this study received no financial support.

\section{References}

1. Goff DC Jr, Lloyd-Jones DM, Bennett G, Coady S, D'Agostino RB, Gibbons R, et al. 2013 ACC/AHA guideline on the assessment of cardiovascular risk: a report of the American College of Cardiology/ American Heart Association Task Force on Practice Guidelines. Circulation 2014; 129: 49-73.

2. Ago T, Sadoshima J. GDF15, a cardioprotective TGF-beta superfamily protein. Circ Res 2006; 98: 294-7.

3. Zhang S, Dai D, Wang X, Zhu H, Jin H, Zhao R, et al. Growth differentiation factor-15 predicts the prognoses of patients with acute coronary syndrome: a meta-analysis. BMC Cardiovasc Disord 2016; 16: 82.

4. Rosner B. Fundamentals of Biostatistics. 7th ed. Boston, Boston: Brooks/Cole, Cengage Learning; 2011. 
5. Matsuzawa $\mathrm{Y}$, Lerman A. Endothelial dysfunction and coronary artery disease: assessment, prognosis, and treatment. Coron Artery Dis 2014; 25: 713-24.

6. Greenland P, Blaha MJ, Budoff MJ, Erbel R, Watson KE. Coronary calcium score and cardiovascular risk. J Am Coll Cardiol 2018; 72 : 434-47.

7. Degrell P, Sorbets E, Feldman LJ, Steg PG, Ducrocq G. Screening for coronary artery disease in asymptomatic individuals: Why and how? Arch Cardiovasc Dis 2015; 108: 675-82.

8. Agatston AS, Janowitz WR, Hildner FJ, Zusmer NR, Viamonte M Jr, Detrano R. Quantification of coronary artery calcium using ultrafast computed tomography. J Am Coll Cardiol 1990; 15: 827-32.

9. Greenland P, LaBree L, Azen SP, Doherty TM, Detrano RC. Coronary artery calcium score combined with Framingham score for risk prediction in asymptomatic individuals. JAMA 2004; 291: 210-5.

10. Polonsky TS, McClelland RL, Jorgensen NW, Bild DE, Burke GL, Guerci $A D$, et al. Coronary artery calcium score and risk classification for coronary heart disease prediction. JAMA 2010; 303: 1610-6.

11. Erbel R, Möhlenkamp S, Moebus S, Schmermund A, Lehmann N, Stang A, et al. Heinz Nixdorf Recall Study Investigative Group. Coronary risk stratification, discrimination, and reclassification improvement based on quantification of subclinical coronary atherosclerosis: the Heinz Nixdorf Recall study. J Am Coll Cardiol 2010; 56: 1397-406.

12. Sangiorgi G, Rumberger JA, Severson A, Edwards WD, Gregoire J, Fitzpatrick LA, et al. Arterial calcification and not lumen stenosis is highly correlated with atherosclerotic plaque burden in humans: a histologic study of 723 coronary artery segments using nondecalcifying methodology. J Am Coll Cardiol 1998; 31: 126-33.

13. Rumberger JA, Simons DB, Fitzpatrick LA, Sheedy PF, Schwartz RS. Coronary artery calcium area by electron-beam computed tomography and coronary atherosclerotic plaque area. A histopathologic correlative study. Circulation 1995; 92: 2157-62.

14. Baumgart D, Schmermund A, Goerge G, Haude M, Ge J, Adamzik M, et al. Comparison of electron beam computed tomography with intracoronary ultrasound and coronary angiography for detection of coronary atherosclerosis. J Am Coll Cardiol 1997; 30: 57-64.

15. van Kempen BJ, Spronk S, Koller MT, Elias-Smale SE, Fleischmann KE, Ikram MA, et al. Comparative effectiveness and cost-effectiveness of computed tomography screening for coronary artery calcium in asymptomatic individuals. J Am Coll Cardiol 2011; 58: 1690-701.

16. Ergun E. Coronary Calcium Scoring. Turkey Radiology Seminars 2013: p. 26-35.

17. Hecht HS, de Siqueira ME, Cham M, Yip R, Narula J, Henschke C, et al. Low- vs. standard-dose coronary artery calcium scanning. Eur Heart J Cardiovasc Imaging 2015; 16: 358-63.
18. Kim KP, Einstein AJ, Berrington de González A. Coronary artery calcification screening: estimated radiation dose and cancer risk. Arch Intern Med 2009; 169: 1188-94.

19. Adela R, Banerjee SK. GDF-15 as a Target and Biomarker for Diabetes and Cardiovascular Diseases: A Translational Prospective. J Diabetes Res 2015; 2015: 490842.

20. Mimeault M, Batra SK. Divergent molecular mechanisms underlying the pleiotropic functions of macrophage inhibitory cytokine-1 in cancer. J Cell Physiol 2010; 224: 626-35.

21. Moore AG, Brown DA, Fairlie WD, Bauskin AR, Brown PK, Munier $\mathrm{ML}$, et al. The transforming growth factor-ss superfamily cytokine macrophage inhibitory cytokine-1 is present in high concentrations in the serum of pregnant women. J Clin Endocrinol Metab 2000; 85: 4781-8.

22. Rohatgi A, Patel P, Das SR, Ayers CR, Khera A, Martinez-Rumayor A, et al. Association of growth differentiation factor-15 with coronary atherosclerosis and mortality in a young, multiethnic population: observations from the Dallas Heart Study. Clin Chem 2012; 58: 172 82.

23. Wollert KC, Kempf T, Wallentin L. Growth Differentiation Factor 15 as a Biomarker in Cardiovascular Disease. Clin Chem 2017; 63: 140151.

24. Lind L, Wallentin L, Kempf T, Tapken H, Quint A, Lindahl B, et al. Growth-differentiation factor-15 is an independent marker of cardiovascular dysfunction and disease in the elderly: results from the Prospective Investigation of the Vasculature in Uppsala Seniors (PIVUS) Study. Eur Heart J 2009; 30: 2346-53.

25. Kempf T, Sinning JM, Quint A, Bickel C, Sinning C, Wild PS, et al. Growth-differentiation factor-15 for risk stratification in patients with stable and unstable coronary heart disease: results from the AtheroGene study. Circ Cardiovasc Genet 2009; 2: 286-92.

26. Schopfer DW, Ku IA, Regan M, Whooley MA. Growth differentiation factor 15 and cardiovascular events in patients with stable ischemic heart disease (The Heart and Soul Study). Am Heart J 2014; 167: 18692.

27. Kempf T, Horn-Wichmann R, Brabant G, Peter T, Allhoff T, Klein G, et al. Circulating concentrations of growth-differentiation factor 15 in apparently healthy elderly individuals and patients with chronic heart failure as assessed by a new immunoradiometric sandwich assay. Clin Chem 2007; 53: 284-91.

28. Wollert KC. Growth-differentiation factor-15 in cardiovascular disease: from bench to bedside, and back. Basic Res Cardiol. 2007; 102: 412-5.

29. Martinez CH, Freeman CM, Nelson JD, Murray S, Wang X, Budoff MJ, et al. GDF-15 plasma levels in chronic obstructive pulmonary disease are associated with subclinical coronary artery disease. Respir Res 2017; 18: 42. 